\title{
Co-detection of Panton-Valentine leukocidin encoding genes and cotrimoxazole resistance in Staphylococcus aureus in Gabon: implications for HIV-patients' care
}

\author{
Christian Kraef ${ }^{1,2}$, Abraham S. Alabi ${ }^{2,3}$, Georg Peters ${ }^{1}$, Karsten Becker ${ }^{1}$, Peter G. Kremsner ${ }^{2,3}$, \\ Elie G. Rossatanga ${ }^{4}$, Alexander Mellmann ${ }^{5}$, Martin P. Grobusch ${ }^{2,3,6}$, Philipp Zanger ${ }^{3,7}$ and \\ Frieder Schaumburg ${ }^{1,2}$ * \\ ${ }^{1}$ Institute of Medical Microbiology, University Hospital Münster, Münster, Germany \\ ${ }^{2}$ Centre de Recherches Médicales de Lambaréné, Albert Schweitzer Hospital, Lambaréné, Gabon \\ ${ }^{3}$ Institut für Tropenmedizin, Eberhard Karls Universität, Deutsches Zentrum für Infektionsforschung, Tübingen, Germany \\ ${ }^{4}$ Centre de Traitement Ambulatoire, Lambaréné, Gabon \\ ${ }^{5}$ Institute of Hygiene, University Hospital Münster, Münster, Germany \\ ${ }^{6}$ Division of Internal Medicine, Center of Tropical Medicine and Travel Medicine, Department of Infectious Diseases, Academic Medical Center, University of \\ Amsterdam, Amsterdam, Netherlands \\ ${ }^{7}$ Institute of Public Health, University Hospital Heidelberg, Heidelberg, Germany
}

\section{Edited by:}

Tzi Bun Ng, The Chinese University of Hong Kong, China

\section{Reviewed by:}

Yuji Morita, Aichi Gakuin University, Japan

Dinesh Sriramulu, Shres Consultancy (Life Sciences), India

\section{*Correspondence:}

Frieder Schaumburg, Institute of Medical Microbiology, University Hospital Münster, Domagkstraße 10, 48149 Münster, Germany

e-mail: frieder.schaumburg@ ukmuenster.de
Patients infected with the human immunodeficiency virus (HIV) are frequently exposed to antimicrobial agents. This might have an impact on the resistance profile, genetic background and virulence factors of colonizing Staphylococcus aureus. Sub-Saharan Africa is considered to be endemic for Panton-Valentine leukocidin (PVL) positive S. aureus which can be associated with skin and soft tissue infections (SSTI). We compared $S$. aureus from nasal and pharyngeal swabs from HIV patients $(n=141)$ and healthy controls $(n=206)$ in Gabon in 2013, and analyzed determinants of colonization with PVL positive isolates in a cross-sectional study. S. aureus isolates were screened for the presence of selected virulence factors (incl. PVL) and were subjected to antimicrobial susceptibility testing and genotyping. In HIV patients, S. aureus was more frequently detected (36.9 vs. 31.6\%) and the isolates were more frequently PVL positive than in healthy controls ( $42.1 \mathrm{vs} .23 .2 \%$ ). The presence of PVL was associated with cotrimoxazole resistance $(O R=25.1, p<0.001)$ and the use of cotrimoxazole was a risk factor for colonization with PVL positive isolates $(\mathrm{OR}=2.5, p=0.06)$. PVL positive isolates were associated with the multilocus sequence types ST15 (OR $=5.6, p<0.001)$ and ST152 (OR $=62.1, p<0.001)$. Participants colonized with PVL positive isolates reported more frequently SSTI in the past compared to carriers of PVL negative isolates (OR=2.7, $p=0.01)$. In conclusion, the novelty of our study is that cotrimoxazole might increase the risk of SSTI in regions where cotrimoxazole resistance is high and associated with PVL. This finding needs to be confirmed in prospective studies.

Keywords: Staphylococcus aureus, Panton-Valentine leukocidin, cotrimoxazole resistance, Africa, HIV

\section{INTRODUCTION}

Staphylococcus aureus is a bacterial pathogen causing a broad range of infections such as skin and soft tissue infections (SSTI), osteomyelitis, pneumonia, and endocarditis (Wertheim et al., 2005). Nasal colonization often precedes invasive infection which is frequently caused by the homolog isolate of the anterior nares (von Eiff et al., 2001). Therefore, nasal colonization rates and molecular characterization of colonizing isolates provide important insights into the risk of $S$. aureus infection in selected human populations. A recent study showed that impairment of the immune system by the human immunodeficiency virus (HIV) increases the risk for S. aureus colonization (Olalekan et al., 2012).

In the present study we compare $S$. aureus from HIV patients and healthy controls as $S$. aureus belongs to the most relevant pathogens of both superficial and invasive infections in Africa (Reddy et al., 2010; Alabi et al., 2013).
Staphylococcus aureus possess an armory of different virulence factors (Otto, 2014b). The Panton-Valentine leukocidin (PVL) is one of the most prevalent virulence determinant in $S$. aureus in sub-Saharan Africa (Schaumburg et al., 2014b). PVL is a bi-component protein toxin that binds to the complement receptor C5a of neutrophils (Spaan etal., 2013). This leukocidin shows strong cytotoxicity on granulocytes and can be associated with severe SSTI (Shallcross et al., 2013). However, its role in pathogenesis in humans remains controversial (Otto, 2014b).

Sub-Saharan Africa suffers from the highest burden of HIV infections in the world; $68 \%$ of all infected persons reside in this region, which only accounts for $12 \%$ of the global population (World Health Organization, 2011). Cotrimoxazole (trimethoprim-sulfamethoxazole) prophylaxis has been shown to lower mortality in HIV infected patients through reduced 
rates of Pneumocystis jirovecii pneumonia, malaria, and bacterial infections (Suthar et al., 2012; Bwakura-Dangarembizi et al., 2014). However, cotrimoxazole is also a broad spectrum antibacterial drug which might impact on S. aureus colonization of HIV patients particularly in long-term use. Cotrimoxazole inhibits the folate biosynthesis by blocking the dihydropteroate synthase (DHPS, sulfamethoxazole) and the dihydrofolate reductase (DHFR, trimethoprim; Lyon and Skurray, 1987). Cotrimoxazole resistance is caused by mutations of the genes encoding the DHPS and/or DHFR or the acquisition of mutated genes through horizontal gene transfer (Lyon and Skurray, 1987). A recent study showed that the DHFR gene $d f r G$ is the major cause of trimethoprim resistance in sub-Saharan Africa (Nurjadi et al., 2014).

In the present study, we (i) compared S. aureus isolates from HIV positive patients with those from healthy controls and (ii) analyzed determinants of colonization with PVL positive isolates. These study objectives are novel as they address for the first time determinants of colonization with PVL positive S. aureus in Africa with a special focus on HIV patients which are frequently exposed to antimicrobial agents.

\section{MATERIALS AND METHODS ETHICAL CLEARANCE}

Ethical approval was obtained from the institutional review board of the "Centre de Recherches Médicales de Lambaréné" in Gabon (CEI 004/2013). Written informed consent was signed by all participants before enrollment.

\section{STUDY DESIGN}

The primary objective of the study was to compare resistance rates and virulence factors of $S$. aureus from HIV patients with those from healthy controls. The secondary objective was to identify risk factors for the colonization with PVL positive isolates.

A recent study from Nigeria showed mean resistance rates to antimicrobial agents (i.e., cotrimoxazole, chloramphenicol, and ciprofloxacin) in S. aureus from HIV positive patients and healthy controls of 51.7 and 23.7\%, respectively (Olalekan et al., 2012). A sample size calculation assuming these mean resistance rates, a power of 0.8 and an alpha of 0.05 gave a total of 53 isolates per group. Assuming an average colonization rate of $37 \%$ in HIV positive patients and $25 \%$ in healthy people yielded a sample size of 143 HIV patients and 212 healthy controls (Shet et al., 2009; Olalekan et al., 2012; Kotpal et al., 2014).

Participants were recruited from the Lambaréné region in Gabon between March and September 2013 if they met the inclusion criteria of (i) providing written informed consent and (ii) age $\geq 18$ years. Subjects with culture confirmed $S$. aureus infections at enrollment were excluded from the final analysis.

Human immunodeficiency virus positive patients attending one of the three out-patient clinics in Lambaréné (Hôpital Albert Schweitzer, Hôpital Régional George Rawiri, Centre de Traitement Ambulatoire) were recruited. Healthy controls were recruited in Lambaréné and surrounding villages.

An HIV test was offered to each participant to verify the HIV status. Demographic data (sex, age), medical history (hospitalization, use of antibiotics in the past 6 months, history of SSTIs), were documented in a standardized questionnaire. The patient's information was derived from records of the personal health care files and interviews.

\section{HIV TEST AND CD4+ CELL COUNT}

Human immunodeficiency virus status was assessed using rapid tests (Determine HIV1/2, Abbott Laboratories, Tokyo, Japan or Vikia, bioMérieux, Marcy l'Etoile, France). T-helper cells [cluster of differentiation four positive cells ( $\mathrm{CD} 4^{+}$cells)] were measured in venous EDTA blood using FACSCount (BD Biosciences, San Jose, CA, USA).

\section{BACTERIAL ISOLATES}

At enrollment, one nasal swab was taken by swabbing gently the mucosa of both anterior nares (including the nasal septum) for few seconds with rotating movements (Schaumburg et al., 2014a). One pharyngeal swab was taken by swabbing the posterior pharyngeal wall with horizontal movements (Schaumburg et al., 2014a). Cotton tips were stored in Amies medium (Transwabs, Medical Wire, Corsham, UK) and cultured within $3 \mathrm{~h}$ for 24-48 h on Columbia blood agar supplemented with aztreonam disks (13 $\mu$ g; Oxoid, Hants, UK; Schaumburg et al., 2014a).

Colonies were tested for positive catalase and agglutination test (Pastorex, Staph-Plus; Bio-Rad Laboratories, Marnes-la-Coquette, France). Species identification and antimicrobial susceptibility test (EUCAST clinical breakpoints) were done using Vitek 2 automated systems (bioMérieux). Species was confirmed by the PCR detection of the thermostable nuclease nuc (Brakstad et al., 1992). Methicillin resistance was confirmed by the PCR detection of the penicillin binding protein $2 \mathrm{a}$ ( $\mathrm{PBP} 2 \mathrm{a}$ ) encoding gene mecA (Becker et al., 2006). All MRSA were screened for the arginine catabolic mobile element (ACME; Diep et al., 2006).

Genes encoding the exfoliative toxins, pyrogenic toxin superantigens (i.e., enterotoxins, toxic shock syndrome toxin) and the Panton-Valentine leukocidin (PVL) were detected by PCR (Lina et al., 1999; Becker et al., 2003).

If a participant was colonized with an identical isolate [based on spa typing (Mellmann et al., 2006)] in the nose and throat, only one isolate was included in the final analysis of resistance rates and virulence factors in order to report non-duplicate isolates only.

\section{GENOTYPING}

All isolates were $S$. aureus protein A $(s p a)$ typed based on the sequence of the hypervariable region of protein A (Mellmann et al., 2006). One isolate of each spa type was subjected to multilocus sequence typing (MLST; Enright et al., 2000) and each MLST sequence type (ST) was assigned to MLST clonal complexes (CC) using eBURST ${ }^{1}$.

\section{STATISTICS}

Colonization was defined as detection of $S$. aureus in the anterior nares and/or pharynx. The $\chi^{2}$ - and Fisher exact tests and logistic regression analyses were used to assess the association of HIV status with demographic characteristics and molecular features of $S$. aureus (e. g. antimicrobial resistance, virulence factors), to estimate odds ratio (OR), the $95 \%$ confidence intervals $(95 \% \mathrm{CI})$

\footnotetext{
${ }^{1}$ http://saureus.mlst.net; hosted at the Imperial College, London
} 
and to adjust for potential confounders. The significance level was set at 0.05 . Mean values are shown $\pm \mathrm{SD}$. Statistical analysis was performed with "R" and the package "epicalc ${ }^{2}$."

To test if statistical associations of the present study could be reproduced, we used a database including 469 non-duplicate isolates (based on spa typing) from healthy, HIV negative, asymptomatic volunteers collected during earlier studies in the Lambaréné region (Schaumburg et al., 2013, 2014a).

\section{RESULTS}

\section{STUDY POPULATION}

In total, 348 participants were recruited; one HIV positive patient was excluded due to a culture confirmed $S$. aureus wound infection at inclusion. Therefore, $141 \mathrm{HIV}$ positive participants and 206 healthy controls were included in the final analysis.

All participants in the HIV group were confirmed to be HIV positive. The median (range) $\mathrm{CD}^{+}$cell count was 166 (2-1374) cells/ $\mu$ l. In total, $49 \mathrm{HIV}$ patients $(34.8 \%)$ were on antiretroviral therapy at the time of inclusion.

In the control group, 68\% $(n=140)$ were HIV negative, $26.9 \%$ $(n=56)$ had a negative test result within the last 3 months but refused a confirmatory test, and $4.9 \%(n=10)$ did not report any test results but refused to be tested for HIV.

\section{COMPARISON OF $S$. aureUS COLONIZATION BETWEEN HIV PATIENTS AND HEALTHY CONTROLS}

On average, participants in both comparison groups were of the same age (Table 1). However, HIV positive subjects were more often female and reported more health care contacts within the past 6 months compared to healthy controls (Table 1). HIV patients significantly used more antimicrobial agents than healthy controls in the past 6 months; such as cotrimoxazole (including cotrimoxazole prophylaxis, 34.0 vs. $1.5 \%$, OR $=33.3,95 \% \mathrm{CI}$ : 11.1-100.0, $p<0.001)$, amoxicillin/clavulanic acid (22.7 vs. $8.3 \%$, $\mathrm{OR}=3.2$, 95\% CI: 1.7-6.7, $p<0.001)$ and ciprofloxacin (9.9 vs. $1.5 \%, \mathrm{OR}=7.7,95 \% \mathrm{CI}: 2.0-50.0, p=0.001)$. No association of S. aureus colonization and $\mathrm{CD} 4^{+}$cell count was detected (data not shown).

${ }^{2}$ http://cran.r-project.org
Staphylococcus aureus colonization was associated with HIV positive participants but did not reach statistical significance (Table 1). This association remained stable when adjusting carriage for history of pyodermatitis, hospitalization and use of antibiotics in the past 6 months (adjusted OR $=0.8,95 \% \mathrm{CI}$ : $0.5-1.2, p=0.3)$.

In total, 125 non-duplicate isolates from HIV patients $(n=56)$ and healthy controls $(n=69)$ were included in the final analysis. Compared to healthy controls, resistance rates of cotrimoxazole were slightly higher in isolates from HIV patients, but did not reach statistical significance (Table 2 ). The distribution of resistance rates of other tested antimicrobial agents was similar in both groups (Table 2). No resistance was detected against clindamycin, linezolid, fosfomycin, fusidic acid, and glycopeptides. For tested virulence factors, PVL was more frequently detected in isolates from HIV patients compared to healthy controls (42.9 vs. $23.2 \%$, Table 2). Genes encoding other virulence factors such as the enterotoxins sea, seg, and sec, the exfoliative toxin a (eta) and the toxic shock syndrome toxin $(t s t)$ were equally distributed among both groups.

The MRSA isolates belonged to spa types t121 $(n=1, \mathrm{ST} 8, \mathrm{PVL}$ positive, ACME positive), t451 ( $n=1, \mathrm{ST} 8$, PVL negative, ACME positive), t1476 ( $n=1$, ST6, PVL negative, ACME negative) and t12858 ( $n=2$, PVL negative, ACME positive). Table 3 shows the distribution of genotypes in all S. aureus isolates from HIV patients and healthy controls.

\section{DETERMINANTS OF COLONIZATION WITH PVL POSITIVE $\boldsymbol{S}$. aureus}

We now addressed potential variables for the colonization with PVL positive $S$. aureus and hypothesized that the use of antimicrobial agents might select for PVL positive isolates. For this purpose, we merged isolates from HIV patients and healthy controls. PVL positive isolates were significantly associated with cotrimoxazole resistance (Table 4). Use of cotrimoxazole was significantly associated with cotrimoxazole resistance $(\mathrm{OR}=3.3,95 \% \mathrm{CI}: 1.0-12.4$, $p=0.03)$. This was in line with an association of cotrimoxazole use and the detection of PVL positive isolates but did not reach statistical significance (Table 4). PVL positive isolates belonged more likely to ST15 and ST152 (Table 4). Cotrimoxazole resistance was strongly associated with ST15 (OR $=18.4,95 \% \mathrm{CI}$ :

Table 1 | Baseline characteristics of the study population.

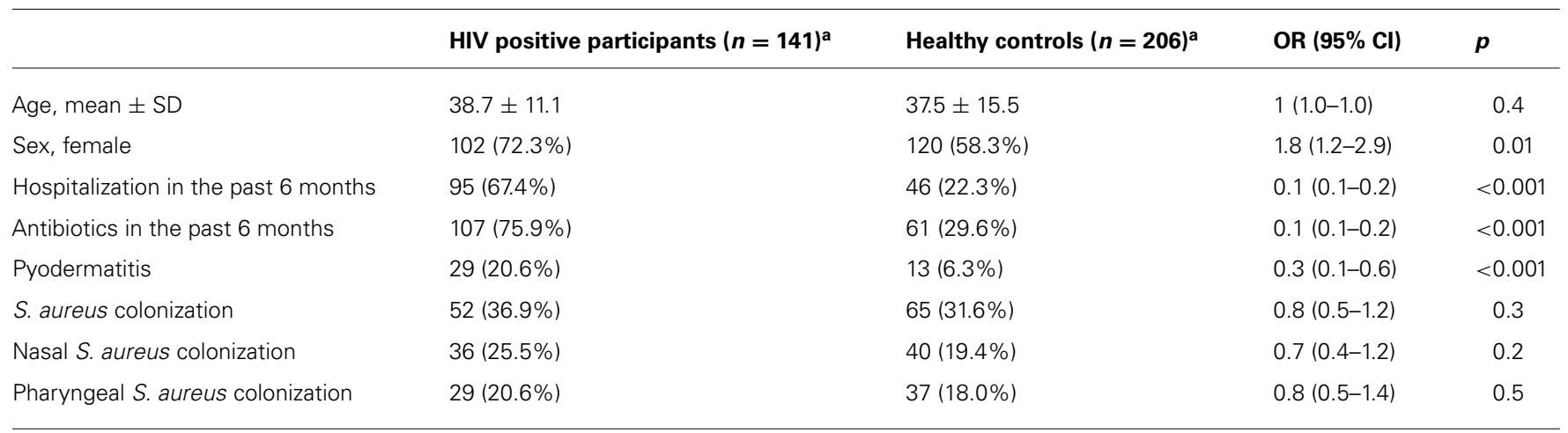

Data are $n(\%)$ of subjects unless otherwise indicated, OR $(95 \% \mathrm{Cl})$ and p-value derived from logistic regression analysis.

a A pharyngeal swab was refused by three HIV positive participants and one control. 
Table 2 | Characteristics of Staphylococcus aureus in HIV patients and healthy controls, Gabon.

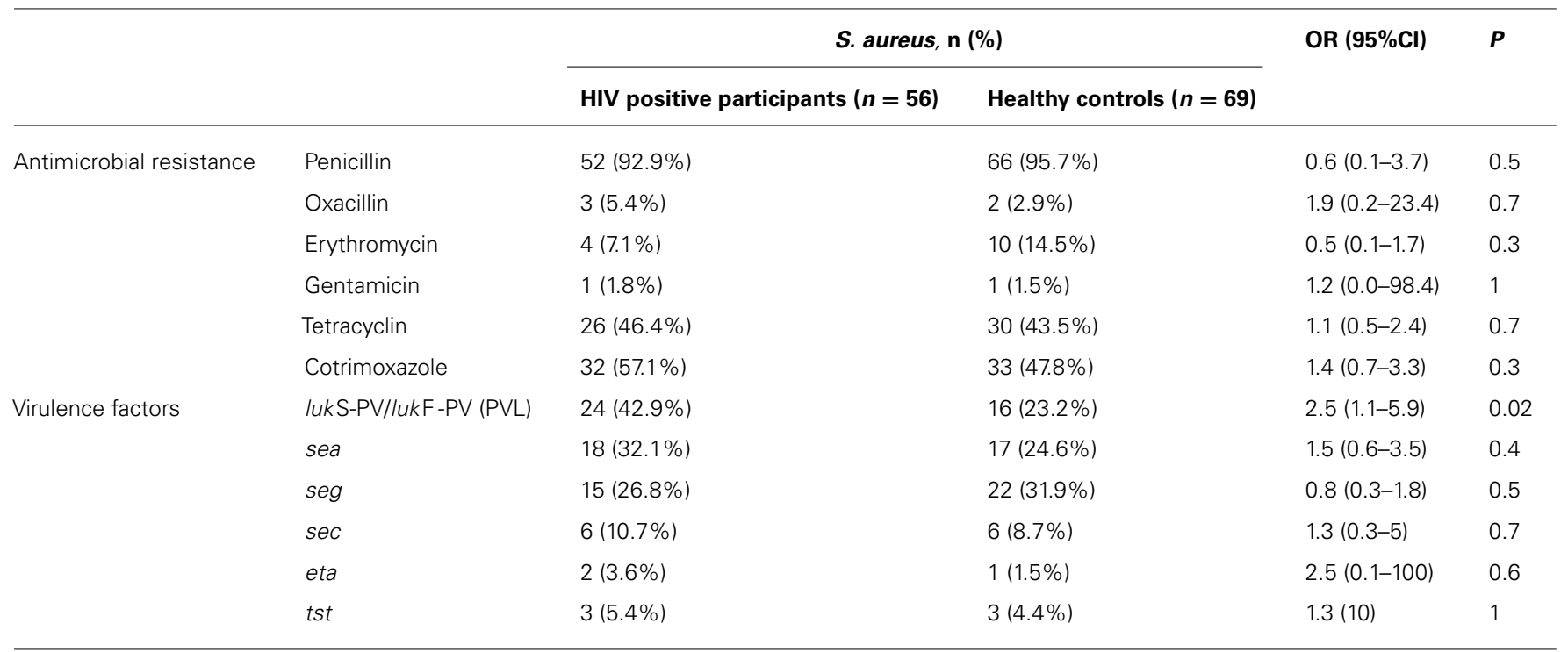

Table 3 | Genotypes of Staphylococcus aureus isolates from HIV patients and healthy controls, in Gabon in 2013.

\begin{tabular}{|c|c|c|c|c|c|c|c|}
\hline CC & ST & S. aureus spa types $(n)$ & Total & S. aureus spa types $(n)$ & Total & OR $(95 \% \mathrm{Cl})^{\mathrm{a}}$ & $p^{a}$ \\
\hline \multirow{7}{*}{ CC5 } & ST5 & t5987 (1) & & t319 (3), t1400 (2), t5987 (4) & & & \\
\hline & ST6 & t701 (1), t1476 (3) & & t304 (1), t701 (1), t1476 (4) & & & \\
\hline & ST9 & t1045 (1) & & t1045 (1) & & & \\
\hline & ST15 & t084 (15), t094 (1), t491 (2), t803 (1) & & t084 (12), t085 (1), t279 (2), t12523 (1) & & & \\
\hline & ST72 & t148 (3) & & t148 (3), t2464 (1), t11108 (1) & & & \\
\hline & ST188 & t189 (1) & & t189 (1) & & & \\
\hline & ST573 & t1579 (1) & & - & & & \\
\hline \multirow[t]{3}{*}{ CC45 } & ST45 & t939 (2) & $7.0 \%$ & t12857 (1) & $4.4 \%$ & $0.6(0.08-3.74)$ & 0.7 \\
\hline & ST508 & t015 (1), t073 (1) & & t12525 (1) & & & \\
\hline & ST2498 & - & & t827 (1) & & & \\
\hline CC101 & ST101 & t056 (1) & $1.8 \%$ & t056 (4), t3165 (1), t4679 (2) & $10.3 \%$ & $6.32(0.76-289.54)$ & 0.07 \\
\hline CC152 & ST152 & t355 (10) & $17.5 \%$ & t355 (8) & $11.8 \%$ & $0.62(0.2-1.9)$ & 0.3 \\
\hline \multirow[t]{4}{*}{ Other } & ST7 & t091 (1) & $8.8 \%$ & - & $1.5 \%$ & $0.31(0.03-2.01)$ & 0.2 \\
\hline & ST88 & t12856 (1) & & t2723 (1) & & & \\
\hline & ST121 & t645 (1) & & - & & & \\
\hline & ST2349 & t026 (1) & & - & & & \\
\hline
\end{tabular}

PVL, positive spa types in bold. ${ }^{\text {a }}$ Comparison of the proportion of clonal complexes (CC). 
Table 4 | Determinants of colonization with PVL positive $S$. aureus.

\begin{tabular}{|c|c|c|c|c|c|c|}
\hline Characteristics & $\begin{array}{l}\text { PVL positive } S \text {. } \\
\text { aureus }(n=40)\end{array}$ & $\begin{array}{l}\text { PVL negative } S \text {. } \\
\text { aureus }(n=85)\end{array}$ & $\begin{array}{l}\text { Crude OR } \\
(95 \% \mathrm{Cl})\end{array}$ & $P$ & $\begin{array}{l}\text { Adjusted } O^{c}{ }^{c} \\
(95 \% \mathrm{Cl})\end{array}$ & $P^{c}$ \\
\hline Cotrimoxazole use $\mathrm{a}^{\mathrm{a}}$ & $10(25.0 \%)$ & $10(11.8 \%)$ & $2.5(0.8-7.4)$ & 0.06 & & \\
\hline Abscess $^{a}$ & $11(27.5 \%)$ & $13(15.3 \%)$ & $2.1(0.8-5.7)$ & 0.1 & & \\
\hline Any SSTI $\left.\right|^{a, b}$ & $20(50.0 \%)$ & $23(27.1 \%)$ & $2.7(1.1-6.3)$ & 0.01 & $2.6(1.2-5.7)$ & 0.02 \\
\hline S. aureus ST15 & $21(52.5 \%)$ & $14(16.5 \%)$ & $5.6(2.2-14.2)$ & $<0.001$ & $5.4(2.3-12.7)$ & $<0.001$ \\
\hline S. aureus ST152 & $17(42.5 \%)$ & $1(1.2 \%)$ & $\begin{array}{l}62.1 \\
(8.5-2584.6)\end{array}$ & $<0.001$ & $\begin{array}{l}65.6 \\
(8.1-532.7)\end{array}$ & $<0.001$ \\
\hline
\end{tabular}

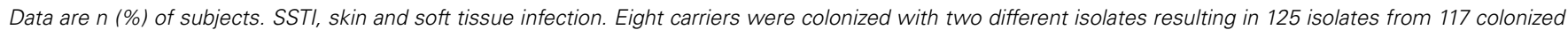
individuals. ${ }^{a}$ in the past 6 months. ${ }^{b}$ any SSTI: wound infection, carbuncle/furuncle, abscess, pyodermatitis, ${ }^{c}$ adjusted for HIV infection.

5.1-99.0, $p<0.001)$ and ST152 (OR $=5.7,95 \%$ CI: $1.5-32.1$, $p=0.004)$. The colonization with either of these genotypes were more common in HIV positive patients compared to healthy controls $(\mathrm{OR}=2.0,95 \% \mathrm{CI}: 0.9-4.4, p=0.06)$ and in participants reporting use of cotrimoxazole $(\mathrm{OR}=2.3,95 \% \mathrm{CI}: 0.8,7.2$, $p=0.08)$.

Of note, participants colonized with PVL positive $S$. aureus reported more frequently any type of SSTIs (pyodermatitis, abscess, furuncle/carbuncle, wound infection) in the past 6 months than those colonized with other isolates (Table 4).

As HIV infection might be a confounder in our dataset we performed a multivariate analysis and adjusted those association for HIV status which were statistically significant in the univariate analysis. HIV infection was a weak confounder of the association between the use of antimicrobials and the detection of PVL positive isolates (Table 4). The associations between PVL and SSTI, cotrimoxazole resistance, ST15 and ST152 remained stable after adjusting for HIV status.

As cotrimoxazole resistant isolates were associated with PVL, we analyzed the association of different exposure variables with the combined outcome "PVL positivity and cotrimoxazole resistance." This combined outcome was also significantly associated with the exposure "use of antimicrobial agents" (OR $=2.4,95 \% \mathrm{CI}$ : $1.1-$ $5.4, p=0.03)$ and "SSTI" (OR $=2.4,95 \% \mathrm{CI}: 1.1-5.3, p=0.03)$ in the past 6 months.

\section{ASSOCIATION OF PVL POSITIVITY AND COTRIMOXAZOLE RESISTANCE IN A RETROSPECTIVE STRAIN COLLECTION}

We now tested if our findings could be reproduced analyzing an already existing strain collection of 469 non-duplicate isolates from healthy, HIV negative, asymptomatic volunteers from earlier studies in the Lambaréné region (Schaumburg et al., 2013, 2014a). Here, we also found strong evidence for an association of cotrimoxazole resistance with the presence of PVL in colonizing $S$. aureus (OR $=5.9,95 \% \mathrm{CI}: 3.7-9.1, p<0.001)$ and with the genotypes ST152 or ST15 (OR = 14.3, 95\%CI: 9.1-25.0, $p<0.001$, Table S1).

\section{DISCUSSION}

Our study found an association of PVL positive isolates with the use of cotrimoxazole and cotrimoxazole resistance. These findings suggest a selection of cotrimoxazole resistant PVL positive $S$. aureus clones through the use of cotrimoxazole. Use of cotrimoxazole might therefore put individuals at higher risk for SSTI in regions where cotrimoxazole resistance is high and associated with PVL. This might be particularly true for HIV patients receiving long term cotrimoxazole prophylaxis.

The higher proportion of females in the HIV group has been reported by others as well and might either reflect a higher risk for HIV infection in females (Olalekan et al., 2012) or gender differences in the overall willingness to participate in research that requires disclosure of HIV status. We detected higher $S$. aureus colonization rates in HIV patients compared to healthy controls, which is in line with a recent report from Nigeria (Olalekan et al., 2012) and a 3-7 fold higher prevalence of HIV infection in adult women compared to men (Karim et al., 2010).

It is well established that the use of antimicrobial agents selects for resistant isolates (Monroe and Polk, 2000; van de Sande-Bruinsma et al., 2008). Therefore, it is not surprising that cotrimoxazole resistance is higher in African HIV patients who are more frequently exposed to cotrimoxazole, as shown in our study. However, cotrimoxazole is also amongst the most frequently used antimicrobial compounds in the general population which explains the high cotrimoxazole resistance in Gabon and other regions of Africa (up to $55 \%$ in colonizing isolates; Schaumburg et al., 2014b).

We showed that the use of cotrimoxazole is a risk factor for colonization with PVL positive and cotrimoxazole resistant ST15 and ST152 isolates. These two lineages are the major PVL positive clones in sub-Saharan Africa (Schaumburg et al., 2014b). Co-occurrence of cotrimoxazole resistance with PVL or with the MLST genotypes ST15 and ST152 was confirmed by reanalyzing published data from healthy asymptomatic carriers in Gabon (Schaumburg et al., 2013, 2014a). It is possible that this finding can 
be extrapolated to other African regions where PVL is associated with cotrimoxazole resistance as well (Breurec et al., 2011).

Altogether, our analysis suggests the following model: cotrimoxazole resistance co-occurs with PVL. The use of cotrimoxazole selects for cotrimoxazole resistant isolates which are frequently PVL positive. The possession of PVL and most likely other virulence factors might pose a risk for SSTI.

However, it remains unclear why cotrimoxazole resistance is co-detected with PVL. One might speculate that genes encoding PVL and cotrimoxazole resistance are carried by the same bacteriophage which has transferred both determinants to the bacterial host genome particularly to $S$. aureus belonging to ST15 and ST152. One might then expect that both genes are located in close proximity to each other. However, to the best of our knowledge, no PVL bacteriophage has been described which also carries antimicrobial resistance genes. Only one novel PVL bacteriophage has been reported which also carried the enterotoxin a gene (sea; Prabhakara et al., 2013). Whole genome sequencing should be performed in future (i) to map the genes of PVL and cotrimoxazole resistance in the bacterial genome and (ii) to assess if both are carried by the same bacteriophage.

Despite these findings we would like to point out that cotrimoxazole prophylaxis should not be discouraged in HIV patients as the benefit has been clearly shown (Suthar et al., 2012; BwakuraDangarembizi et al., 2014), and as clinical implications of PVL are still controversial (Otto, 2014b). The implication of our study on HIV patients' care is to sensitize physicians for the counselling on and the early detection of SSTI in patients receiving cotrimoxazole prophylaxis. A higher risk of SSTI in these patients might be possible as our analysis revealed an association of PVL positive isolates with a history of SSTI in the past 6 months that was independent of the HIV status. In addition, a strong association of PVL with SSTIs was also recently shown by a large meta-analysis (Shallcross et al., 2013).

However, PVL is not the only virulence factor which can be associated with SSTIs. Strong tissue toxicity has also been shown for the $\alpha$-toxin or the phenol-soluble modulins (Becker et al., 2014; Otto, 2014a).

Our study is limited by our small sample size and an unknown HIV status of $10(4.9 \%)$ participants. We believe that the risk of miss-including HIV positive patients in the control group is low as the HIV prevalence was low in Gabon in 2009 (5.2\%, declining trend) compared to South and East Africa (World Health Organization, 2011). In addition these participants were not followed by any of the HIV clinics in Lambaréné. Second, the setting of the recruitment was not the same for HIV positive patients (clinics) and controls (community setting). As only healthy controls should be included, we were not able to recruit them in the hospital setting. Third, the cross-sectional study design cannot detect the causality of high prevalence of PVL positive isolates. We only are able to identify potential factors causing a selection of PVL positive isolates which might be addressed in future prospective studies.

In conclusion, cotrimoxazole use was associated with PVL positive and cotrimoxazole-resistant S. aureus. Therefore, cotrimoxazole prophylaxis may put HIV patients at risk of SSTIs in regions where cotrimoxazole resistance is high and associated with PVL.

\section{ACKNOWLEDGMENTS}

We thank Harry Kaba, Damayanti Kaiser and Heike Rengbers for the brilliant technical assistance and the staff of the Albert Schweitzer Hospital in Lambaréné for the kind collaboration. This work was supported by the Deutsche Forschungsgemeinschaft (EI 247/8-1).

\section{SUPPLEMENTARY MATERIAL}

The Supplementary Material for this article can be found online at: http://www.frontiersin.org/journal/10.3389/fmicb.2015.00060/ abstract

\section{REFERENCES}

Alabi, A., Frielinghaus, L., Kaba, H., Kosters, K., Huson, M. A., Kahl, B., et al. (2013). Retrospective analysis of antimicrobial resistance and bacterial spectrum of infection in Gabon, Central Africa. BMC Infect. Dis. 13:455. doi: 10.1186/14712334-13-455

Becker, K., Friedrich, A. W., Lubritz, G., Weilert, M., Peters, G., and von Eiff, C. (2003). Prevalence of genes encoding pyrogenic toxin superantigens and exfoliative toxins among strains of Staphylococcus aureus isolated from blood and nasal specimens. J. Clin. Microbiol. 41, 1434-1439. doi: 10.1128/JCM.41.4.1434-1439.2003

Becker, K., Pagnier, I., Schuhen, B., Wenzelburger, F., Friedrich, A. W., Kipp, F., et al. (2006). Does nasal cocolonization by methicillin-resistant coagulase-negative staphylococci and methicillin-susceptible Staphylococcus aureus strains occur frequently enough to represent a risk of false-positive methicillin-resistant $S$. aureus determinations by molecular methods? J. Clin. Microbiol. 44, 229-231. doi: 10.1128/JCM.44.1.229-231.2006

Becker, R. E., Berube, B. J., Sampedro, G. R., DeDent, A. C., and Bubeck Wardenburg, J. (2014). Tissue-specific patterning of host innate immune responses by Staphylococcus aureus a-toxin. J. Innate Immun. 6, 619-631. doi: $10.1159 / 000360006$

Brakstad, O. G., Aasbakk, K., and Maeland, J. A. (1992). Detection of Staphylococcus aureus by polymerase chain reaction amplification of the nuc gene. J. Clin. Microbiol. 30, 1654-1660.

Breurec, S., Fall, C., Pouillot, R., Boisier, P., Brisse, S., Diene-Sarr, F., et al. (2011). Epidemiology of methicillin-susceptible Staphylococcus aureus lineages in five major African towns: high prevalence of Panton-Valentine leukocidin genes. Clin. Microbiol. Infect. 17, 633-63. doi: 10.1111/j.1469-0691.2010.03320.x

Bwakura-Dangarembizi, M., Kendall, L., Bakeera-Kitaka, S., Nahirya-Ntege, P., Keishanyu, R., Nathoo, K., et al. (2014). A randomized trial of prolonged cotrimoxazole in HIV-Infected children in Africa. N. Engl. J. Med. 370, 41-53. doi: 10.1056/NEJMoa1214901

Diep, B. A., Gill, S. R., Chang, R. F., Phan, T. H., Chen, J. H., Davidson, M. G., et al. (2006). Complete genome sequence of USA300, an epidemic clone of community-acquired meticillin-resistant Staphylococcus aureus. Lancet 367, 731-739. doi: 10.1016/S0140-6736(06)68231-7

Enright, M. C., Day, N. P., Davies, C. E., Peacock, S. J., and Spratt, B. G. (2000). Multilocus sequence typing for characterization of methicillin-resistant and methicillin-susceptible clones of Staphylococcus aureus. J. Clin. Microbiol. 38, 1008-1015.

Karim, Q. A., Sibeko, S., and Baxter, C. (2010). Preventing HIV infection in women: a global health imperative. Clin. Infect. Dis. 50, S122-S129. doi: 10.1086/651483

Kotpal, R., Krishna, P. S., Bhalla, P., Dewan, R., and Kaur, R. (2014). Incidence and risk factors of nasal carriage of Staphylococcus aureus in HIV-infected individuals in comparison to HIV-uninfected individuals: a case-control study. J. Int. Assoc. Provid. AIDS Care doi: 10.1177/2325957414554005 [Epub ahead of print].

Lina, G., Piemont, Y., Godail-Gamot, F., Bes, M., Peter, M. O., Gauduchon, V., et al. (1999). Involvement of Panton-Valentine leukocidin-producing Staphylococcus aureus in primary skin infections and pneumonia. Clin. Infect. Dis. 29, 1128-1132. doi: $10.1086 / 313461$

Lyon, B. R., and Skurray, R. (1987). Antimicrobial resistance of Staphylococcus aureus: genetic basis. Microbiol. Rev. 51, 88-134. 
Mellmann, A., Friedrich, A. W., Rosenkotter, N., Rothganger, J., Karch, H., Reintjes, R., et al. (2006). Automated DNA sequence-based early warning system for the detection of methicillin-resistant Staphylococcus aureus outbreaks. PLoS Med. 3:e33. doi: 10.1371/journal.pmed. 0030033

Monroe, S., and Polk, R. (2000). Antimicrobial use and bacterial resistance. Curr. Opin. Microbiol. 3, 496-501. doi: 10.1016/S1369-5274(00) 00129-6

Nurjadi, D., Olalekan, A. O., Layer, F., Shittu, A. O., Alabi, A., Ghebremedhin, B., et al. (2014). Emergence of trimethoprim resistance gene dfrG in Staphylococcus aureus causing human infection and colonization in sub-Saharan Africa and its import to Europe. J. Antimicrob. Chemother. 69, 2361-2368. doi: 10.1093/jac/ dku174

Olalekan, A. O., Schaumburg, F., Nurjadi, D., Dike, A. E., Ojurongbe, O., Kolawole, D. O., etal. (2012). Clonal expansion accounts for an excess of antimicrobial resistance in Staphylococcus aureus colonising HIV-positive individuals in Lagos, Nigeria. Int. J. Antimicrob. Agents 40, 268-272. doi: 10.1016/j.ijantimicag.2012.05.016

Otto, M. (2014a). Phenol-soluble modulins. Int. J. Med. Microbiol. 304, 164-169. doi: 10.1016/j.ijmm.2013.11.019

Otto, M. (2014b). Staphylococcus aureus toxins. Curr. Opin. Microbiol. 17, 32-37. doi: 10.1016/j.mib.2013.11.004

Prabhakara, S., Khedkar, S., Shambat, S. M., Srinivasan, R., Basu, A., NorrbyTeglund, A., et al. (2013). Genome sequencing unveils a novel sea EnterotoxinCarrying PVL phage in Staphylococcus aureus ST772 from India. PLoS ONE 8:e60013. doi: 10.1371/journal.pone.0060013

Reddy, E. A., Shaw, A. V., and Crump, J. A. (2010). Community-acquired bloodstream infections in Africa: a systematic review and meta-analysis. Lancet Infect. Dis. 10, 417-432. doi: 10.1016/S1473-3099(10)70072-4

Schaumburg, F., Alabi, A. S., Mombo-Ngoma, G., Kaba, H., Zoleko, R. M., Diop, D. A., et al. (2014a). Transmission of Staphylococcus aureus between mothers and infants in an African setting. Clin. Microbiol. Infect. 20, O390-O396. doi: 10.1111/1469-0691.12417

Schaumburg, F., Alabi, A. S., Peters, G., and Becker, K. (2014b). New epidemiology of Staphylococcus aureus infection from Africa. Clin. Microbiol. Infect. 20, 589-596. doi: 10.1111/1469-0691.12690

Schaumburg, F., Biallas, B., Feugap, E. N., Alabi, A. S., Mordmüller, B., Kremsner, P. G., et al. (2013). Carriage of encapsulated bacteria in Gabonese children with sickle cell anaemia. Clin. Microbiol. Infect. 19, 235-241. doi: 10.1111/j.14690691.2012.03771.x

Shallcross, L. J., Fragaszy, E., Johnson, A. M., and Hayward, A. C. (2013). The role of the Panton-Valentine leucocidin toxin in staphylococcal disease: a systematic review and meta-analysis. Lancet Infect. Dis. 13, 43-54. doi: 10.1016/S14733099(12)70238-4
Shet, A., Mathema, B., Mediavilla, J. R., Kishii, K., Mehandru, S., Jeane-Pierre, P., et al. (2009). Colonization and subsequent skin and soft tissue infection due to Methicillin-Resistant Staphylococcus aureus in a cohort of otherwise healthy adults infected with HIV Type 1. J. Infect. Dis. 200, 88-93. doi: 10.1086/599315

Spaan, A. N., Henry, T., van-Rooijen, W. J., Perret, M., Badiou, C., Aerts, P. C., et al. (2013). The staphylococcal toxin Panton-Valentine leukocidin targets human C5a receptors. Cell Host Microbe 13, 584-594. doi: 10.1016/j.chom.2013.04.006

Suthar, A. B., Granich, R., Mermin, J., and Van Rie, A. (2012). Effect of cotrimoxazole on mortality in HIV-infected adults on antiretroviral therapy: a systematic review and meta-analysis. Bull. World Health Organ. 90, 128C-138C. doi: 10.2471/BLT.11.093260

van de Sande-Bruinsma, N., Grundmann, H., Verloo, D., Tiemersma, E., Monen, J., Goossens, H., et al. (2008). Antimicrobial drug use and resistance in Europe. Emerg. Infect. Dis. 14, 1722-1730. doi: 10.3201/eid1411.070467

von Eiff, C., Becker, K., Machka, K., Stammer, H., and Peters, G. (2001). Nasal carriage as a source of Staphylococcus aureus bacteremia. N. Engl. J. Med. 344, 11-16. doi: 10.1056/NEJM200101043440102

Wertheim, H. F., Melles, D. C., Vos, M. C., van Leeuwen, W., van Belkum, A., Verbrugh, H. A., et al. (2005). The role of nasal carriage in Staphylococcus aureus infections. Lancet Infect. Dis. 5, 751-762. doi: 10.1016/S1473-3099(05)70295-4

World Health Organization. (2011). Global HIV/AIDS Response: Epidemic Update and Health Sector Progress Towards Universal Access. Progress Report (Geneva: World Health Organization).

Conflict of Interest Statement: The authors declare that the research was conducted in the absence of any commercial or financial relationships that could be construed as a potential conflict of interest.

Received: 20 November 2014; accepted: 17 January 2015; published online: 05 February 2015.

Citation: Kraef C, Alabi AS, Peters G, Becker K, Kremsner PG, Rossatanga EG, Mellmann A, Grobusch MP, Zanger P and Schaumburg F (2015) Co-detection of Panton-Valentine leukocidin encoding genes and cotrimoxazole resistance in Staphylococcus aureus in Gabon: implications for HIV-patients' care. Front. Microbiol. 6:60. doi: $10.3389 /$ fmicb.2015.00060

This article was submitted to Antimicrobials, Resistance and Chemotherapy, a section of the journal Frontiers in Microbiology.

Copyright (C) 2015 Kraef, Alabi, Peters, Becker, Kremsner, Rossatanga, Mellmann, Grobusch, Zanger and Schaumburg. This is an open-access article distributed under the terms of the Creative Commons Attribution License (CC BY). The use, distribution or reproduction in other forums is permitted, provided the original author(s) or licensor are credited and that the original publication in this journal is cited, in accordance with accepted academic practice. No use, distribution or reproduction is permitted which does not comply with these terms. 